\title{
I Luoghi della Memoria: temi e prospettive
}

\author{
di Alberto Gagliardo
}

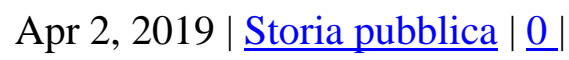

\section{Appunti dal Convegno di Rocca delle Caminate - 7 dicembre 2018}

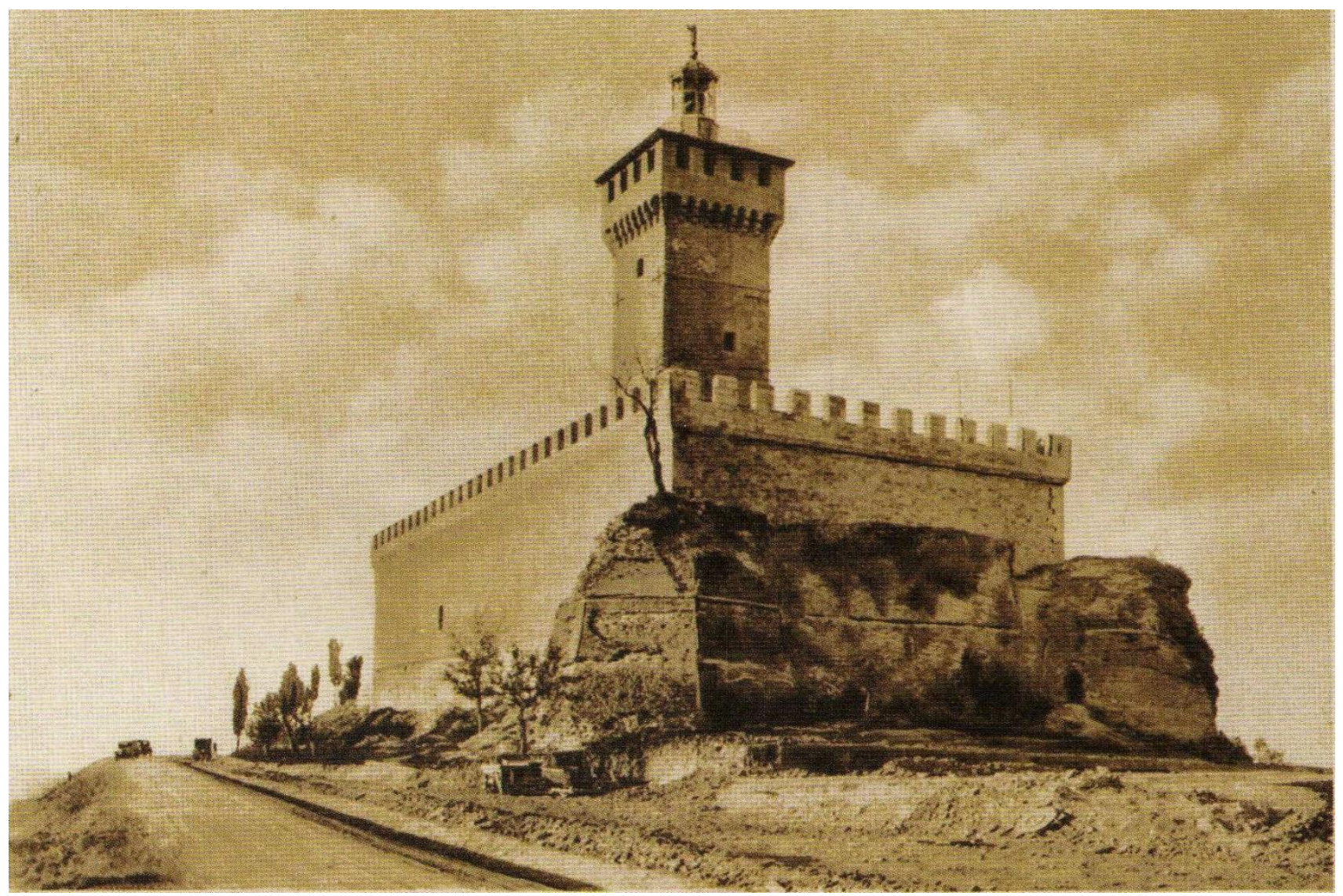

Rocca delle Carminate dopo il restauro, $\underline{\text { Pubblico dominio, }}$ Collegamento

\begin{abstract}
Il 7 dicembre 2018 si è svolto a Rocca delle Caminate (FC) il convegno di studi I Luoghi della Memoria: temi e prospettive, promosso dalla Rete regionale degli Istituti storici emilianoromagnoli, dal Coordinamento provinciale per i luoghi della memoria di Forlì-Cesena, dalla Rotta culturale europea ATRIUM e dai Comuni di Meldola e di Predappio. Nell'articolo si riportano i tratti più significativi e interessanti delle relazioni di quella giornata.
\end{abstract}

\section{Cosa sono i luoghi della memoria}

È stato Pierre Nora (Les Lieux de Mémoire, Paris, Gallimard, 3 voll., 1984-1992) a formulare, per primo, una definizione di "luogo della memoria" come «una unità significativa, d'ordine materiale o ideale, che la volontà degli uomini o il lavorio del tempo ha reso un elemento simbolico di una qualche comunità. [...] Il luogo della memoria ha come scopo fornire al visitatore, al passante, il quadro autentico e concreto di un fatto storico. Rende visibile ciò che non lo è: la storia [...] e unisce in un unico campo due discipline: la storia appunto e la geografia». L'espressione coniata da 
Nora e il suo pensiero di storico sono caratteristici della tradizione storica francese degli «Annales» come l'autore dichiarava sin dall'eloquente sottotitolo: «itinéraires géographiques»

In ambito italiano l'espressione viene introdotta da Mario Isnenghi (a cura di, I luoghi della memoria, Roma-Bari, Laterza, 3 voll., 1996-1997), che individua una serie di "luoghi" reali e simbolici, monumentali o legati al paesaggio, la cui nozione è incrociata con quella di memoria e di identità nazionale.

Tra memoria e conoscenza storica: perché valorizzare i luoghi della memoria

In estrema sintesi, pur nelle specificità di interessi e risultati, ciò che accomuna i lavori di questi studiosi è la consapevolezza che con l'espressione "luoghi della memoria" si rimanda sempre ad una pluralità di situazioni e significati, oltre che di possibilità di approccio.

E plurime sono innanzitutto le modalità di valorizzazione e di utilizzo dei "luoghi di memoria": un luogo può infatti rimandare a una storia/memoria dominante o a più storie/memorie, proprio perché in passato può aver assunto rilevanze e funzioni diverse, a seconda del periodo e dell'uso che se ne è fatto. Inoltre un luogo può presentarsi in una dimensione fluida e articolata, anche perché il ricordo dei testimoni offre un ulteriore materiale di riflessione, ricco e insidioso al tempo stesso.

Infine, un luogo può essere inserito a pieno titolo in un itinerario di conoscenza storica, a patto di essere indagato con gli strumenti e le modalità della ricerca che collocano le fonti e la memoria tra gli elementi indispensabili per la ricostruzione di precise vicende e situazioni.

Ma soprattutto il concetto di "luogo di memoria" chiama in causa i valori fondamentali della comunità che lo ospita, lo frequenta e lo fa vivere, dal momento che, oltre ad esprimere la fiducia collettiva nei valori di educazione, istruzione, conoscenza sottesi alla sua istituzione, contemporaneamente ne custodisce e forgia i valori di cittadinanza posti a fondamento di quella stessa società.

\section{Il convegno del 7 dicembre}

E proprio su questi temi il 7 dicembre 2018 si è svolto a Rocca delle Caminate (FC) il convegno di studi I Luoghi della Memoria: temi e prospettive, promosso dalla Rete regionale degli Istituti storici emiliano-romagnoli, dal Coordinamento provinciale per i luoghi della memoria di Forlì-Cesena, dalla Rotta culturale europea ATRIUM e dai Comuni di Meldola e di Predappio.

Al Convegno hanno inoltre offerto la propria collaborazione l'Istituto nazionale "Ferruccio Parri”, 1'Associazione Paesaggi della memoria, "E-Review. Rivista degli Istituti storici dell'EmiliaRomagna in Rete", e "Clionet - Per un senso del tempo e dei luoghi. Rivista di Public History".

Esso, infine, ha ricevuto il sostegno da Ser.In.Ar. (Servizi integrati d'area) e i patrocini della Regione Emilia-Romagna e della Provincia di Forlì-Cesena. 
Un programma di lavori davvero ricco e nutrito che ha visto, dopo i saluti istituzionali di Massimo Mezzetti (Assessore alla Cultura della Regione Emilia-Romagna), di Gian Luca Zattini (Sindaco di Meldola) e di Giorgio Frassineti (Sindaco di Predappio), una serie di interventi di sicuro interesse. Un programma che, pur privo della relazione introduttiva sul Concetto di luogo di memoria e sulla sua evoluzione di Alberto Cavaglion (assente per un impedimento dell'ultimo momento), cornice teorica per le successive riflessioni, è riuscito tuttavia a far emergere un quadro concettuale di riferimento, a partire dalle singole esperienze raccontate o dalle specifiche problematiche affrontate in ciascuno degli interventi.

\section{Mirco Carrattieri (Istituto storico di Reggio Emilia, Museo di Montefiorino) \\ I Musei storici italiani sul '900: un settore in fermento}

Il quadro tratteggiato dal relatore ha proposto, anche attraverso una puntuale ricostruzione dei principali riferimenti normativi, una panoramica dei Musei storici italiani dedicati al Novecento che ha spesso operato confronti con le corrispondenti esperienze straniere, delineando un settore piuttosto dinamico e in vivace fermento in cui, accanto alla resistenza di impostazioni più tradizionali, si vanno imponendo forme e linguaggi nuovi per la messa in mostra della storia. Dalla disamina è emerso come oggi le nuove tecnologie siano caratterizzate sia dalla capacità di valorizzare al massimo la ricerca storica-giacché favoriscono la lettura contemporanea di dati e documenti- sia dall'opportunità di un'interazione ricca e immediata tra fruitore e oggetto. Per questo esse paiono senz'altro gli strumenti più idonei alla restituzione di una ricerca storica mirata sui luoghi della memoria. Di tale tendenza l'esempio più illuminante è senz'altro rappresentato dal Museo del Novecento M9, di recentissima apertura a Mestre, che, come recita il sito, «pone la multimedialità e l'interattività al servizio della narrazione storica». In questa stessa direzione si collocano anche altri esempi presentati nell'ambito delle relazioni esposte nel corso della giornata di studi.

\section{Carlo De Maria (Università di Bologna, Istituto storico di Forlì-Cesena)}

Luoghi della memoria e didattica della storia

L'intervento ha proposto una riflessione sui temi dei luoghi di memoria e della didattica della storia, cogliendoli in una relazione dialettica e individuando anche nella public history un possibile punto di contatto e di reciproca azione feconda fra i due territori. Essendo infatti quest'ultima la «storia narrata, rappresentata e comunicata per un pubblico ampio, di non specialisti, di non addetti ai lavori», il cui «obiettivo è promuovere l'utilità della storia nella società, favorire una crescita civile e di consapevolezza nel corpo sociale; contribuire a dare un senso allo scorrere del tempo e maggiore spessore storico al nostro sguardo sui luoghi che attraversiamo», la public history diventa una naturale alleata tanto della didattica della storia quanto del racconto dei luoghi di memoria, condividendo con entrambi gli obiettivi di rigore, chiarezza, comprensibilità, divulgazione.

\section{Mirco Zanoni (Istituto Cervi)}

\section{Note a margine di un censimento regionale sui luoghi di memoria}

Il discorso ha preso le mosse dal censimento regionale sui luoghi della memoria incardinando la riflessione sull'area emiliano-romagnola con stimolanti riflessioni sulla peculiarità della situazione locale, caratterizzata, simultaneamente, da un forte sensibilità delle Istituzioni e da una non altrettanto forte capacità di "fare sistema" da parte delle singole realtà territoriali. Ad esempio, il relatore ha osservato come, a fronte di un investimento finanziario pubblico senza eguali in Italia e in virtù del quale moltissime classi scolastiche effettuano viaggi sui luoghi della memoria di vari Paesi europei- da quest'anno anche su quelli dell'identità europea, VAE- tuttavia l'Emilia-Romagna non riesce ad attrarre sul proprio territorio le corrispettive esperienze estere di viaggi della memoria 
cioè ad essere meta di viaggi della memoria da altre regioni italiane o dall'estero, pur vantando uno straordinario patrimonio di storie, luoghi e memorie del ' 900.

\section{Elena Pirazzoli (ricercatrice indipendente)}

I luoghi della discordia. Il perpetuo presente del dissonant heritage

Marcatamente connotato da un'analisi comparativa tra Italia ed altri Paesi l'intervento della relatrice è risultato incentrato su una considerazione di partenza: spesso un "luogo di memoria" è un patrimonio il cui simbolo ereditato dal passato non è più condiviso dalla comunità che lo ospita; oppure è un patrimonio attorno al quale vi è un disaccordo nella comunità sul significato da attribuirgli e sull'interpretazione che deve esserne data. Ma anche in questo caso la relatrice ha saputo far dialogare il quadro teorico generale con le esperienze locali, prendendo a spunto proprio dalla storia di Rocca delle Caminate e dalla sua "discordante eredità", come testimoniano le polemiche di recente ravvivate a proposito delle proposte di riaccendere "faro del Duce".

Questo caso specifico, proprio come un case study- ha mostrato come la memoria, nel fissarsi in un luogo, possa assumere una pluralità di forme che, attraverso differenti modalità di comunicazione $\mathrm{e}$ linguaggi (monumentalizzazione, conservazione, simbolizzazione, ecc.), determinano gradi più o meno complessi di riconoscibilità e fruizione da parte del pubblico, rendendo necessari approcci ogni volta diversi. Per cui, restando al caso in questione, non è tanto importante riaccendere o non riaccendere il faro della Rocca, bensì capire, sapere e spiegare che cosa significhi davvero riaccenderlo o meno. Dunque si tratta non di cancellare una memoria storica, benché scomoda e urticante, sedimentata nel luogo, ma di recuperarla, rendendola nuovamente parte della comunità grazie ad un altro significato.

\section{Claudio Silingardi (Istituto nazionale Ferruccio Parri)}

Governance dei luoghi: contrasti e tensioni

Il relatore ha affrontato il delicato tema evidenziando tensioni sia in senso orizzontale, tra "centro e periferia", sia in senso verticale, tra "alto e basso" delle gerarchie istituzionali. Da questa relazione è emerso con chiarezza che se la Regione Emilia-Romagna, attraverso la legge .3/2016 (Memoria del Novecento. Promozione e sostegno delle attività di valorizzazione della Storia del Novecento), da un lato si è dotata di uno strumento unico nel panorama nazionale per promuovere studio, ricerca, attività didattica, conservazione e valorizzazione della conoscenza storica della contemporaneità, dall'altro appare necessario ancora un lungo lavoro perché i soggetti coinvolti da tale investimento sappiano creare strategie condivise e di ampio respiro, cioè sappiano muoversi in sinergia su disegni di ampio respiro. Bisognerebbe uscire dalla logica che troppo spesso ha guidato, e a volte guida ancora, il rapporto tra le Amministrazioni pubbliche e le Istituzioni preposte alla tutela della memoria e dei suoi luoghi. Infatti se prima tale rapporto era fondato su una presunta o presumibile "affinità valoriale-culturale", quasi ineludibile conditio sine qua non, oggi questo rapporto va riqualificato, ricalibrato a partire dal saper accettare la sfida dei nuovi tempi e delle nuove sensibilità, per poi muoversi nella direzione della solidità e dunque della credibilità scientifica di una proposta offerta per la tutela e la valorizzazione dei luoghi di memoria stessi.

\section{I lavori del pomeriggio}

\section{Fausto Ciuffi (Fondazione Villa Emma)}

Passato e presente nella memoria di un luogo. Tra ricerca e progettazione: il caso Nonantola. Nel pomeriggio i lavori sono ripresi con l'intervento di Fausto Ciuffi, il quale ha proposto una esperienza che ha assunto il valore di "studio di caso". La storia di Villa Emma risulta infatti paradigmatica della complessità con cui ci si deve confrontare per valorizzare una vicenda così ricca e unica nel panorama europeo, prima ancora che italiano. 
Essa ci mostra come tra luogo e memoria le relazioni siano molteplici e mutevoli, perché ogni luogo sta nel tempo e dunque può essere attraversato e abitato da più storie. Come ci dimostra il caso di Villa Emma, un luogo può contenere una o più storie, con cui esso può interagire, confondersi, trasformarsi. Vale a dire uno stesso luogo può resistere diversamente al tempo e finire preservato o dimenticato; o ancora un luogo può toccare diverse culture e sensibilità e, di conseguenza, modificarsi esso stesso. Infine un luogo può parlare a intere comunità e aggregazioni sociali, così come può sollecitare coscienze o curiosità individuali. Di tutta questa stratificata complessità deve farsi carico una proposta di riqualificazione e valorizzazione culturale che voglia essere all'altezza del proprio compito.

\section{Vladimiro Flamigni (Istituto storico di Forli-Cesena)}

\section{Da Tavolicci ai Percorsi della solidarietà in guerra}

Analoghe osservazioni ha proposto il relatore quando ha raccontato le esperienze in corso nel territorio forlivese che mettono in evidenza la grande potenzialità didattica di questi luoghi di memoria, sia nel loro aspetto fisico e materiale (i sentieri appenninici, la casa dell'eccidio di Tavolicci), sia in quello virtuale per le potenzialità offerte dalle nuove tecnologie informatiche. In quest'ultimo caso l'esempio è venuto dall'illustrazione del portale in via di costruzione, ma già navigabile, dell'Istituto Storico Forlivese: https://percorsisolidarieta.istorecofc.it/. Anzi proprio l'utilizzo delle nuove tecnologie e dei nuovi media, guidato da competenze e saperi maturati in lunghi periodi di studio, può costituire un antidoto a quell'eccesso di pessima informazione dilagante nella Rete, dove la logica orizzontale dell" "uno vale uno", produce la morte della competenza (Tom Nichols, The death of expertise, 2017), sicura anticamera di oscurantismo e illiberalità.

\section{Luca Alessandrini (Istituto per la storia e le memorie del 900 "Parri” Emilia-Romagna)} Extra monasterium servientes. La nascita e la vita del Museo per la Memoria di Ustica. Anche in questo intervento il relatore ha svolto un ragionamento alto sul rapporto tra conservazione della memoria e sua diffusione nel tessuto civile, ancora una volta partendo da un caso specifico e molto particolare. Tra i tanti elementi messi in luce dal relatore, anche quello del rapporto tra gli aspetti "scientifico ed estetico" della messa in mostra (non secondario in un allestimento museale costruito da un affermato artista internazionale, Christian Boltanski) che ha offerto preziose occasioni di riflessione. Infatti attribuire ai luoghi di memoria un potenziale, un valore formativo e orientativo, cioè considerarli come generatori di identità, implica un utilizzo corretto di precise categorie interpretative e di adeguati scenari storiografici. Significa collocare vicende ed elaborazioni collettive all'interno di relazioni più ampie, ispirate da un senso di responsabilità verso il passato. Vuol dire considerare il presente come terreno di dialogo e di confronto, combattere il rischio delle facili generalizzazioni.

In sintesi, nell'intervento di Alessandrini sono sembrate riecheggiare le ormai celebri parole di Pierre Nora: «Memoria e storia non sono affatto sinonimi, tutto le oppone. La memoria è sempre in evoluzione, soggetta a tutte le utilizzazioni e manipolazioni; la storia è la ricostruzione, sempre problematica e incompleta, di ciò che non c'è più. Carica di sentimenti e di magia, la memoria si nutre di ricordi sfumati; la storia, in quanto operazione intellettuale e laicizzante, richiede analisi e discorso critico. La memoria colloca il ricordo nell'ambito del sacro, la storia lo stana e lo rende prosaico».

\section{Elena Monicelli (Scuola di Pace di Monte Sole)}

"Se solo fosse così semplice": sfide e opportunità di un discorso sul presente nei luoghi di memoria Nella sua relazione Elena Monicelli ha invece sviluppato il delicato intreccio tra la riflessione storica e il rapporto che essa inevitabilmente intreccia con il presente. Un luogo di memoria ha bisogno infatti di un lavoro di organizzazione storico e scientifico preciso, che parta dall'individuazione del luogo, per procedere, con la raccolta della documentazione, all'allestimento 
di un percorso materiale (musei, casa-museo, monumenti) o virtuale (Internet), e di una struttura testuale valida che permetta alle tracce di memoria presenti in quel luogo particolare di poter essere lette e condivise dalla compagine sociale nel proprio tempo.

Tavola Rotonda: Mirco Carrattieri (Paesaggi della Memoria), Pierluigi Castagnetti (Fondazione Fossoli), Davide Drei (Sindaco di Forlì), Paolo Lucchi (Sindaco di Cesena), Albertina Soliani (Istituto Cervi)

La tavola rotonda conclusiva, coordinata da Mario Proli (Comune di Forli), ha ripercorso i nodi problematici più significativi, offrendo, grazie anche all'apporto fornito dalla partecipazione del sindaco di Forlì Davide Drei, utili occasioni di confronto scaturite da approcci e prospettive diverse.

Dagli interventi è comunque emerso, con grande evidenza, come i luoghi di memoria abbiano enormi potenzialità (non ultime quelle turistica e, dunque, economica), fra le quali quella didattica è sicuramente fra le più incisive ed efficaci, dal momento che, partire da un territorio del quale gli studenti, specie i più piccoli, possono esperire una diretta conoscenza, corrisponde a precise esigenze formative e psicologiche, poiché mette in atto meccanismi importanti di riflessione, non solo cognitiva, operando il passaggio dal "vicino" al "lontano", dal familiare all'ignoto, dal concreto all'astratto (De Maria).

Ed è proprio su questo terreno che, se tutti i soggetti coinvolti sapranno cogliere l'ampiezza e l'importanza della sfida, si gioca una cruciale partita civile, prima ancora che culturale.

\section{Osservazioni conclusive}

Come si desume da questa disamina, sia pure sintetica, è stata senz'altro rispettata l'idea centrale del convegno che muoveva proprio da un'esigenza concreta di riflessione e di rilancio in merito al lavoro svolto negli ultimi decenni in alcuni luoghi della memoria del territorio romagnolo (tra $i$ quali, Tavolicci, Fragheto, Pieve di Rivoschio e Ca' Cornio), unita alla volontà però di costruire un'occasione di confronto di rilevanza regionale e nazionale.

Anche la scelta del luogo, Rocca delle Caminate, non è stata casuale, giacché al tema dei luoghi della memoria si intende collegare anche quello di un "Centro di documentazione della solidarietà europea", che si vorrebbe istituire proprio qui.

Così pure sono stati rispettati gli obiettivi della giornata di studi: conoscenza dei termini di discussione teorica in corso (i luoghi della memoria tra ricerca storica, didattica della storia e public history); analisi delle principali esperienze operative (buone pratiche e criticità nella gestione dei luoghi della memoria, dei musei diffusi e dei networks); ricognizione delle nuove soluzioni museografiche e valutazione della loro realizzabilità concreta (rapporto tra beni culturali, innovazione e digitale, ovvero i musei di ultima generazione); confronto fra esperienze e punti di vista differenti (i politici, gli amministratori, i tecnici) di quanti si occupano di progettare il recupero dei luoghi della memoria, per dare indicazioni utili dal punto di vista pratico-operativo a operatori e fruitori.

Infine, proprio dalla conoscenza degli aspetti teorici coinvolti, dalla consapevolezza delle principali esperienze operative e dalla ricognizione delle nuove soluzioni proposte o ancora da sviluppare, nasce un'opportunità di rinnovamento anche per la didattica della storia e la sua trasmissione alle nuove generazioni. 
Per questo il Convegno si configurava anche come Corso di aggiornamento, registrato sulla piattaforma ministeriale SOFIA, aperto agli insegnanti dei vari ordini e gradi d'istruzione. Perché è la scuola il luogo privilegiato dove si formano le capacità di leggere e di dialogare con i luoghi di memoria, dove si educano le sensibilità di quanti quei luoghi visiteranno e ne tramanderanno il testimone, è soprattutto da lì che dovranno venire le curiosità e le energie che li terranno vivi e attivi. 